Supporting Information

\title{
Controlling the Lattice Parameters of Gold Nanoparticle FCC Crystals with Duplex DNA Linkers
}

\author{
Haley D. Hill ${ }^{\dagger 1}$, Robert J. Macfarlane ${ }^{\dagger 1}$, Andrew J. Senesi ${ }^{1}$, Byeongdu Lee ${ }^{2}$, Sung \\ Yong Park ${ }^{3}$, Chad A. Mirkin ${ }^{* 1}$ \\ ${ }^{1}$ Department of Chemistry and International Institute for Nanotechnology, Northwestern \\ University, 2145 Sheridan Rd., Evanston, IL 60208-3113. \\ ${ }^{2}$ X-ray Science Division, Advanced Photon Source, Argonne National Laboratory, 9700 S. Cass \\ Ave., Argonne, IL 60439. \\ ${ }^{3}$ Department of Biostatistics and Computational Biology, University of Rochester, 601 Elmwood \\ Ave., Rochester, New York 14642, USA
}

\section{Detailed Materials and Methods:}

DNA functionalized gold nanoparticles (DNA-AuNPs) were prepared according to literature procedures using AuNPs obtained from BBInternational with an average diameter of $10.3 \pm 1 \mathrm{~nm}$. All oligonucleotides (Scheme 1) were synthesized on an Expidite DNA synthesizer and purified by HPLC. Briefly, hexyl-thiol terminated oligonucleotides (5'-HS-A ${ }_{10^{-}}$ AAGACGAATATTTAACAA-3') were added to the AuNPs at a ratio of 4 nmol per $1 \mathrm{~mL}$ of AuNPs. The $\mathrm{A}_{10}$ section allows for DNA strand flexibility, while the thiol is used to attach the DNA to the AuNP surface. These DNA-AuNP solutions were then slowly salted to final concentrations of $0.5 \mathrm{M} \mathrm{NaCl}, 10 \mathrm{mM}$ sodium phosphate and $0.01 \% \mathrm{wt} / \mathrm{vol} \mathrm{SDS}$ and subsequently washed five times (centrifugation at 15k RPM for $40 \mathrm{~min}$, followed by removal of supernatant and resuspension in Nanopure 18.2 $\mathrm{M} \Omega \mathrm{H}_{2} \mathrm{O}$ ) to eliminate all unbound DNA from the DNAAuNPs. UV-Vis absorption spectra for particles and DNA strands were measured on a Cary 100 and converted to concentration using Beer's Law and known extinction coefficients.

Each linker strand contained a $3^{\prime}$ terminus complimentary to the Au-bound DNA strands (5'-TTGTTAAATATTCGTCTT- 3') and a self-complimentary 5' GCGC terminus. The 5' and 3' 
ends of the oligonucleotide were connected by a sequence of repeated thymine bases flanked on either end by an adenine "wobble" base to allow DNA strand flexibility. The thymine sections were 13, 26, 39 and 52 bases in length — a fifth linker was synthesized without any additional thymines and with only one wobble base (X-Linker). 13, 26, 39 and 52 base poly-A sequences were synthesized to hybridize with these poly-T sequences to give the system rigidity. The duplex spacers containing the poly-A sequences and poly-T linkers were hybridized by heating them to $55{ }^{\circ} \mathrm{C}$ and slowly cooling to room temperature in $0.5 \mathrm{M} \mathrm{NaCl}, 10 \mathrm{mM} \mathrm{H}_{\mathrm{x}} \mathrm{PO}_{4} 0.01 \%$ $\mathrm{wt} / \mathrm{vol}$ SDS. These linker sequences were then added to the solutions of DNA-AuNPs in a 40 to 1 ratio, transferred to quartz capillaries and heated to $45^{\circ} \mathrm{C}$. The temperature was lowered at a rate of $0.1{ }^{\circ} \mathrm{C} /$ minute until the sample reached $35^{\circ} \mathrm{C}$, at which time it was imaged with smallangle x-ray scattering (SAXS).

The SAXS experiments were performed at the Dupont-Northwestern-Dow Collaborative Access Team (DND-CAT) Sector 5 at Argonne National Laboratory. Aqueous samples were placed in $2 \mathrm{~mm}$ quartz capillaries and inserted into the beamline equipped with a temperature controlling system; the temperature was controlled within $0.1{ }^{\circ} \mathrm{C}$ resolution. Two sets of slits were used to define and collimate a $0.3 \times 0.3 \mathrm{~mm}^{2}$ x-ray beam (wavelength $=1.03 \AA$ ) and a pinhole was used to remove parasitic scattering. Scattered radiation was detected with a CCD camera. The 2D scattering data were azimuthally averaged, and the resulting $1 \mathrm{D}$ profiles of scattered intensity as a function of scattering angle, $2 \theta$, were transformed into profiles of scattered intensity as a function of scattering vector, $\mathrm{q}(=4 \pi \sin \theta / \lambda)$, using silver behenate as a standard. Dark current subtraction and absorption corrections were applied to normalize the data. Although the scattering from the buffer and capillary are weak compared to the scattering from the gold nanoparticles, buffer data was still used to reduce the data to scattering patterns representative of the particles alone. 


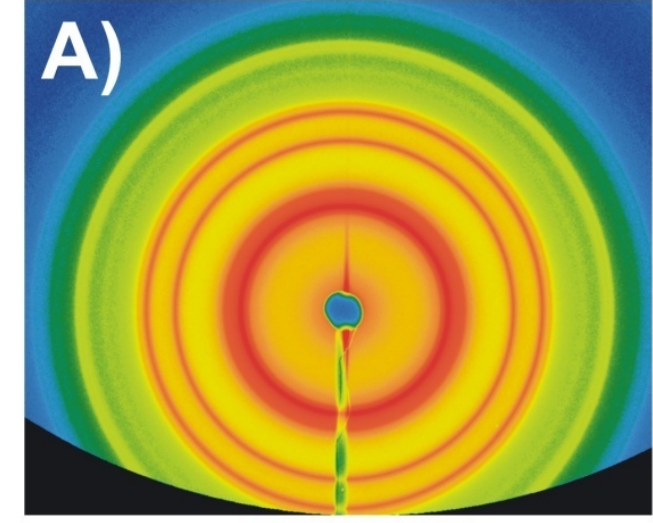

X-Linker

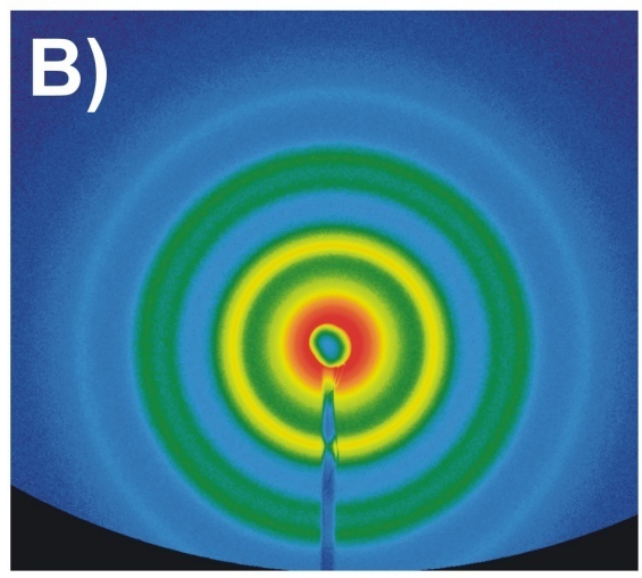

13-Linker

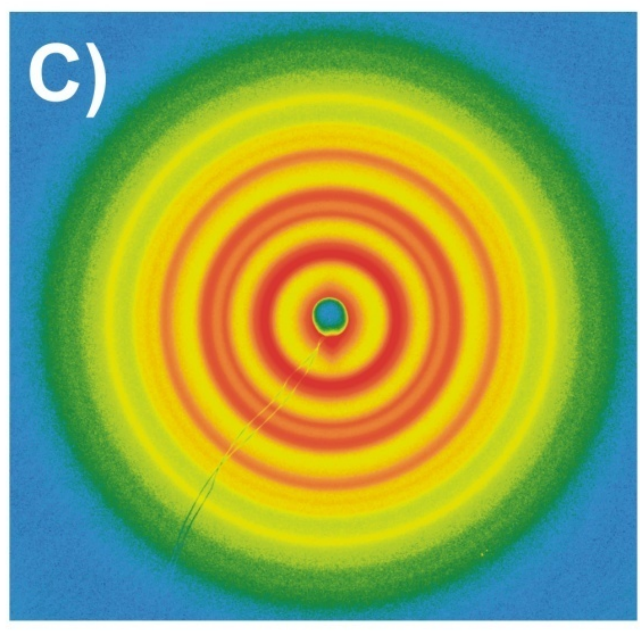

26-Linker

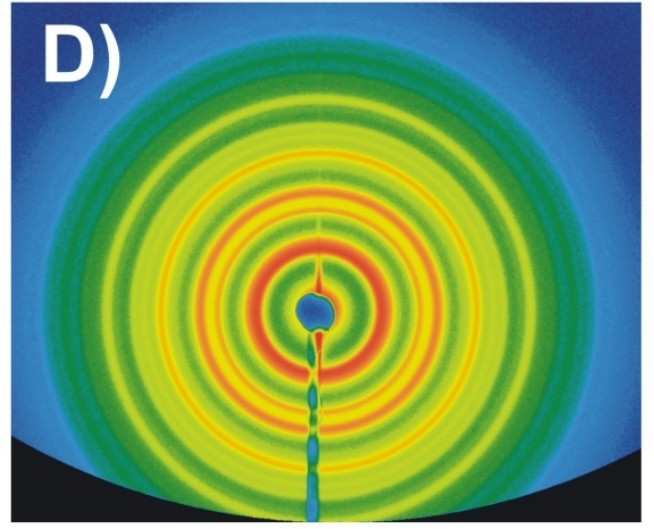

39-Linker

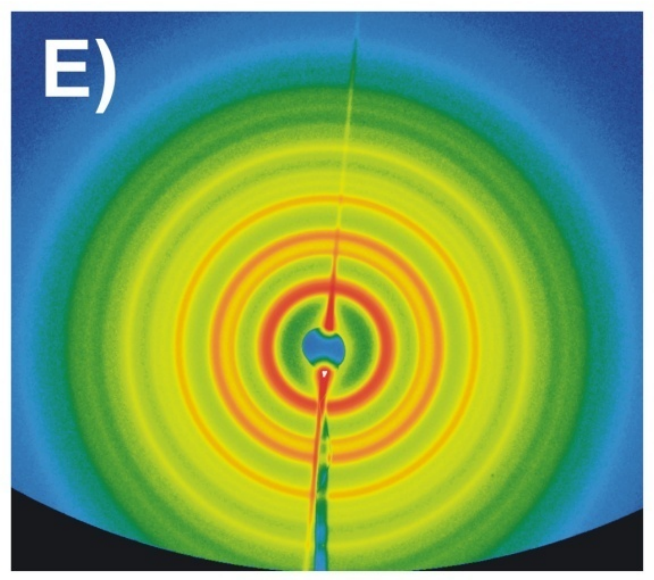

52-Linker

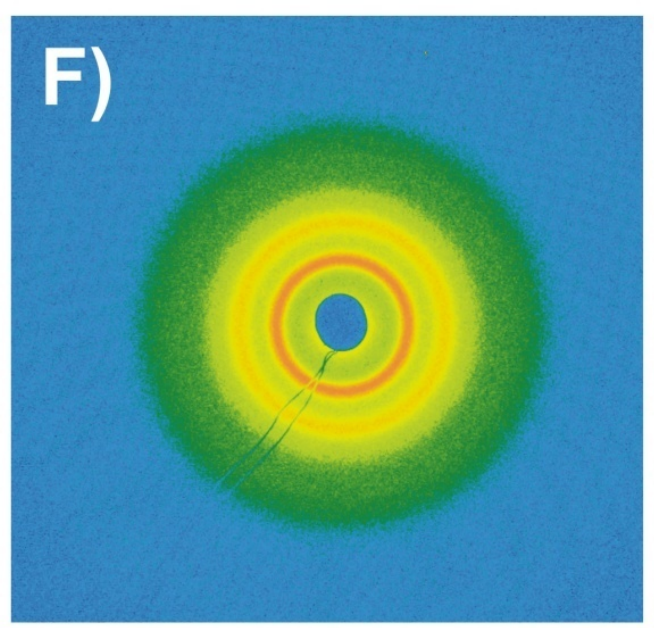

65-Linker

Supporting Figure 1. 2-Dimensional SAXS Images for All Linkers. 

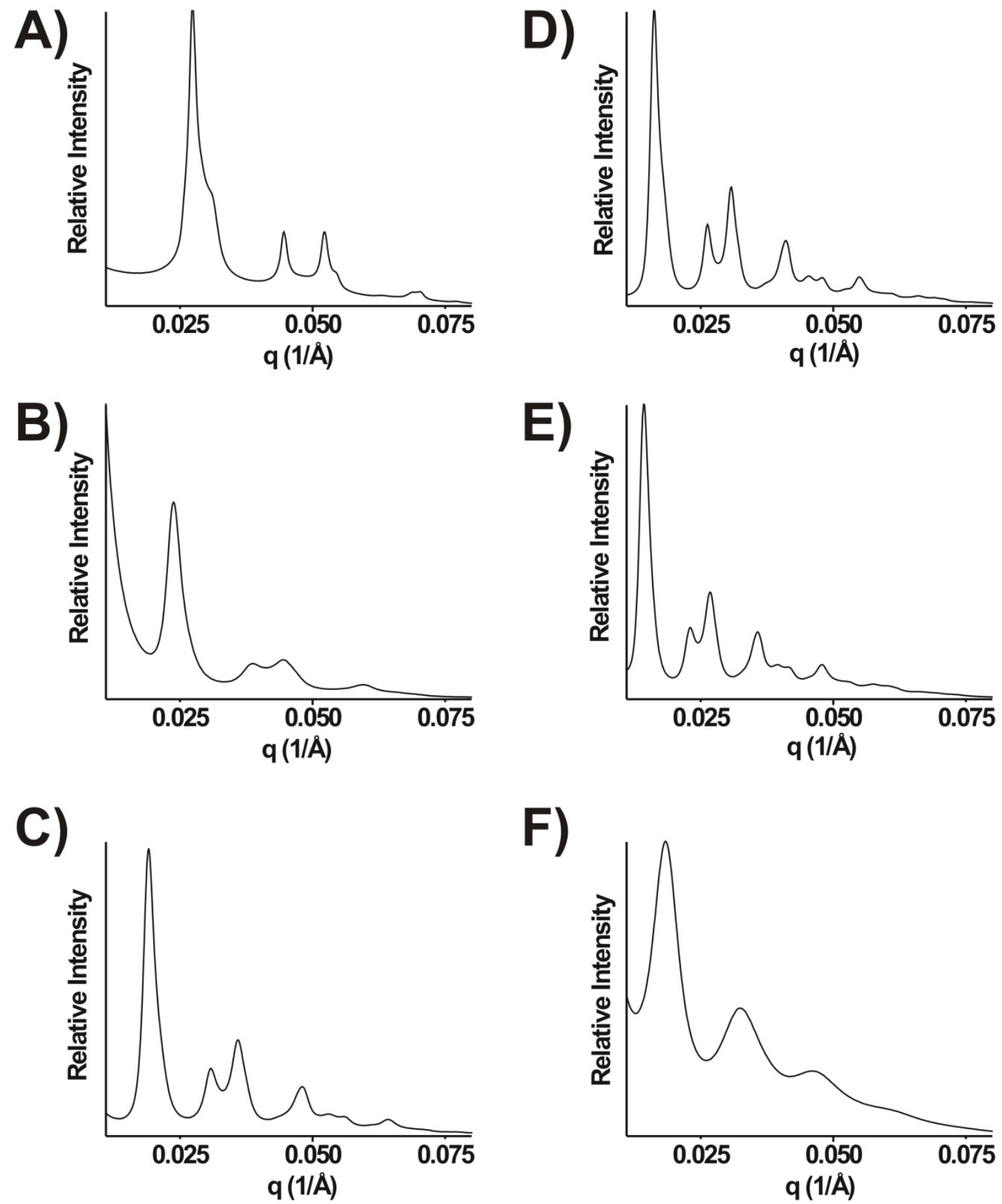

Supporting Figure 2. 1-Dimensional SAXS Images for All Linkers. A) X-Linker, B) 13Linker, C) 26-Linker, D) 39-Linker, E) 52-Linker, F) 65-Linker. 